\title{
Performance limit of AOA-based localization using MIMO-OFDM channel state information
}

\author{
Tanee Demeechai ${ }^{*}$ and Pratana Kukieattikool
}

\begin{abstract}
Wireless communication networks are increasingly based on the ubiquitous multiple-input multiple-output orthogonal frequency division multiplexing (MIMO-OFDM) modulation scheme. Their channel state information is generally obtained each time by a base station receiver as soon as a data packet is successfully received from a mobile device. As it has been shown recently that the MIMO-OFDM channel state information can be used for angle of arrival-based localization, this paper presents a theoretical investigation of the localization performance. The method of computing the Cramer-Rao lower bound, which represents the performance of a minimum variance unbiased estimator, is presented and then used for insightful investigation purposes by means of inspecting the viability of the system requirements and the design properties.
\end{abstract}

Keywords: Localization, Angle of arrival, MIMO-OFDM, Channel state information, Multipath propagation, Cramer-Rao lower bound

\section{Introduction}

Wireless localization is currently a research topic of primary interest in the field of wireless communications due to providing a large variety of location-based services for increasingly ubiquitous smart mobile devices. For wireless localization, a method requiring hardware of just an existing wireless network is of particular interest, because no extra hardware is required for both the infrastructure and the mobile devices (MDs). Unfortunately, although many studies have investigated wireless localization [1-5], it is not straightforward to apply their results without requiring dedicated hardware or affecting the network protocol structure.

A few localization methods requiring hardware of just an existing wireless network have been proposed in the literature [6-9] and implemented commercially. Most of them [6-8] adopt fingerprint matching as the basic scheme of location determination. In this scheme, prior to the localization operation, algorithm parameters are determined by a training process. The parameters are

\footnotetext{
*Correspondence: tanee.demeechai@nectec.or.th

National Electronics and Computer Technology Center, Phahonyothin Road, 12120 Pathum Thani, Thailand
}

determined in principle as a representation of the characteristics of the observation data associated with a number of MD reference locations. In the localization operation, the location is determined as a linear combination of the reference locations, based on the observation data and the trained parameters. One major drawback of this scheme is that the training process is usually a costly measurement process. In addition, because the radio wave propagation characteristics that characterize the observation data are sensitive to the environment, the training process will be required every time the environment of the localization region has changed.

We realize that the angle of arrival (AOA) estimation $[10,11]$ is an interesting study that can be applied for localization. Recently, it has been shown that multipleinput multiple-output (MIMO) orthogonal frequency division multiplexing (OFDM) channel state information (CSI) may be used as the observation data for localization based on AOA [9]. For localization based on AOA, it generally requires that direct radio paths between the $\mathrm{MD}$ and the base stations (BSs) are available sufficiently for triangulation. However, using MIMO-OFDM CSI as the observation data for localization based on AOA is of particular interest here, due to the following reasons. 
AOA-based localization does not require a costly training process. In addition, the MIMO-OFDM technique is currently the top air interface technology prevalently employed for very high-speed wireless networks.

The purpose of this paper is to investigate the performance characteristics of AOA-based localization methods that employ the MIMO-OFDM CSI as the observation data. This may be done by using the Cramer-Rao lower bound (CRLB), which is the variance of the estimation error of a minimum variance unbiased estimator (MVUE), as it has been successfully used to investigate theoretical performance characteristics for a number of related estimation problems [12-14]. In this paper, a method of computing the CRLB in general for the AOA-based localization that uses MIMO-OFDM CSI as the observation data is presented. Numerical results are then obtained for a specific case detailing about the geometry and placement of the BS antenna arrays and the localization region. The results are discussed, respecting the impact of radio propagation characteristics and system infrastructures on the average system performance and providing fundamental insights on the system requirements and design principles.

\section{System and observation models}

Channel frequency response (CFR) for a used OFDM subcarrier is a necessary information for demodulation of data carried by that subcarrier. Conventionally, the transmitter needs to facilitate CFR estimation at the receiver by provision of pilot bits [15]. It is then reasonable to assume that once a receiver can successfully detect the data carried by the used OFDM subcarriers, it can also provide the CFR for the set of used OFDM subcarriers, i.e., the CSI, at no overhead. For localization, the CSI may be obtained, based on either uplink or downlink transmission, for sending to a localization processor. In this paper, we are only interested in localization with processor residing in the network infrastructure to avoid burdening the generally resource-limited MDs. In this case, uplink CSI estimated by a BS receiver may be sent to the processor at low cost via an existing non-radio connection. On the other hand, sending the downlink CSI estimated by a MD receiver to the processor inevitably requires some radio resource that can be costly. Reducing the cost of sending the downlink CSI to the processor may be done with a chunk-based scheme [16-18], where an average magnitude of CFR for a block of contiguous subcarriers is sent instead of the complex values of the CFR of all subcarriers within the block. However, AOA-based localization requires the CFR in complex form [9]. Therefore, strictly following [16-18] may not be appropriate for our problem. In this regard, we consider that a similar chunkbased scheme that sends an average complex CFR for the block instead of the average magnitude may be adopted for AOA-based localization. This is because the average CFR obtained by this scheme is essentially same as that obtained by a conventional subcarrier-based scheme with the same total bandwidth but larger subcarrier spacing. However, this scheme therefore provides CFR with less details along the frequency axis. Accordingly, the processor will perform worse on separating the direct path from reflection paths, leading eventually to lower performance.

As using the uplink CSI for the localization processor residing in the infrastructure is less cumbersome compared with using the downlink CSI, we assume that the location of a MD is determined by processing the CSI obtained by the MIMO-OFDM receivers of all BSs upon successful reception of a data packet from that MD. Assume that each BS employs an array of antennas, with the spacing between them on the order of half wavelength. Then, the CFR for the $q$-th BS respecting the $k$-th subcarrier and $m$-th antenna can be expressed by [9]

$$
H_{q, k, m}=\sum_{n=0}^{N_{\mathrm{P}}-1} g_{q, n} e^{-j\left(2 \pi t_{q, n} k \Delta+\phi_{q, n, m}\right)}+w_{q, k, m},
$$

where $N_{\mathrm{P}}$ is the number of paths in the radio wave propagation model, $\Delta$ is the OFDM subcarrier spacing, $g_{q, n}$ is the complex gain with magnitude representing the strength of the $n$-th path for the $q$-th BS, $t_{q, n}$ is the propagation delay associated with the $n$-th path between the MD antenna and the center of the antenna array of the $q$-th BS and subtracted by a constant delay specific to the $q$-th BS, $\phi_{q, n, m}, \forall m$ are the phase terms obeying $\sum_{\forall m} \phi_{q, n, m}=0$ and sharing a relationship that depends on the $q$-th BS antenna array geometry and the AOA to the array from the $n$-th path, and $w_{q, k, m}$ is the estimation error that is assumed to behave as additive white Gaussian noise (AWGN). Assume that the terms in (1) have been sorted in ascending order of $t_{q, n}$, i.e., $t_{q, n_{1}} \leq t_{q, n_{2}}$ if $n_{1} \leq n_{2}$. In addition, it is required that direct path from the MD antenna to the $\mathrm{BS}$ antenna array exists as the shortest path. Thus, it is the relationship between $\phi_{q, 0, m}$, $\forall m$ that contains the AOA-based location information of the MD.

We have also assumed that the OFDM time synchronizer and provision of the guard interval that houses the cyclic prefix of the OFDM symbol are perfect on their roles. Hence, the following can be deduced in subsequent. First, by perfect provision of the guard interval, the maximum delay spread is not greater than the guard interval length, i.e., $t_{q, N_{\mathrm{P}}-1}-t_{q, 0} \leq t_{\mathrm{g}}$, where $t_{\mathrm{g}}$ is the guard interval length. As a result, letting the $l$-th OFDM symbol excluding cyclic prefix begin arriving at the receiver at time instant $t_{\mathrm{d}}$, we can say that the contribution of the $(l-1)$-th OFDM symbol arriving at the receiver ends accordingly at time instant $t_{\mathrm{d}}-t_{\mathrm{m}}$, where $t_{\mathrm{m}} \geq 0$ and $t_{\mathrm{m}}=t_{\mathrm{g}}-\left(t_{q, N_{\mathrm{P}}-1}-t_{q, 0}\right)$. Then, by perfect time 
synchronization, the receiver should be able to avoid intersymbol interference (ISI) by beginning the demodulation of the $l$-th OFDM symbol at $t_{0}$ with $t_{\mathrm{d}}-t_{\mathrm{m}} \leq t_{0} \leq t_{\mathrm{d}}$. Then, by virtue of the cyclic prefix, the orthogonality between the subcarriers is still preserved, and the direct path manifests itself to the synchronized demodulator as having delay of $t_{q, 0}=t_{\mathrm{d}}-t_{0}$. Note that a good practice of OFDM time synchronization is to have $t_{0}$ close to $t_{\mathrm{d}}$. Accordingly, $t_{q, 0}$ is usually a small non-negative number compared with the guard interval length.

\section{CRLB computation}

The computation of CRLB can be based on the following theoretical assumptions. The number of antennas which are included in the array corresponds to $M_{q}$ for the $q$-th BS. Then, the observation data from the $q$-th BS may be expressed in a matrix form as

$$
\mathbf{H}_{q, k}=\mathbf{S}_{q, k}+\mathbf{W}_{q, k}
$$

where $\mathbf{W}_{q, k}=\left[\begin{array}{llll}w_{q, k, 1} & w_{q, k, 2} & \cdots & w_{q, k, M_{q}}\end{array}\right]^{\mathrm{T}}$, with $(\cdot)^{\mathrm{T}}$ denoting the matrix transpose, $\mathbf{S}_{q, k}=\mathbf{A}_{q} \mathbf{G}_{q, 0} \mathbf{R}_{q, 0}^{k}$, and we have used the following notations.

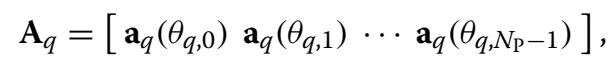

where

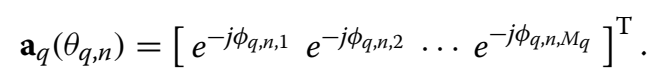

Note that $\mathbf{a}_{q}(\cdot)$ is a vector-valued function that depends on the $q$-th BS antenna array geometry, and $\theta_{q, n}$ denotes the AOA to the array from the $n$-th radio path. In addition, we have assumed that an AOA can be represented by a single number, and hence, two-dimensional localization is implied.

$$
\begin{aligned}
& \mathbf{G}_{q, i}=\operatorname{diag}\left\{g_{q, n}\right\}_{n=i}^{N_{\mathrm{P}}-1}, \\
& \mathbf{R}_{q, i}^{k}=\left[e^{-j \omega_{q, i} k} e^{-j \omega_{q, i+1} k} \cdots e^{-j \omega_{q, N_{\mathrm{P}}-1} k}\right]^{\mathrm{T}},
\end{aligned}
$$

where $\omega_{q, n}=2 \pi t_{q, n} \Delta$. Denote the number of involved BSs as $Q$. Then, since $\theta_{q, 0}, 1 \leq q \leq Q$ are uniquely determined by the MD location given the geometry and placement of all the BS antenna arrays, we may define the row vector of independent parameters for the observation data as

$$
\mathbf{u}=\left[\begin{array}{llllll}
x_{0} & y_{0} & \mathbf{v}_{1} & \mathbf{v}_{2} & \cdots & \mathbf{v}_{Q}
\end{array}\right],
$$

where $x_{0}$ and $y_{0}$ represent the MD location in a Cartesian coordinate system, and

$$
\mathbf{v}_{q}=\left[\Omega_{q} \Re\left(\mathbf{g}_{q}\right) \Im\left(\mathbf{g}_{q}\right) \Theta_{q}\right],
$$

with $\Omega_{q}=\left[\omega_{q, 0} \omega_{q, 1} \cdots \omega_{q, N_{\mathrm{P}}-1}\right], \mathbf{g}_{q}=\left[g_{q, 0} g_{q, 1} \cdots\right.$ $\left.g_{q, N_{\mathrm{P}}-1}\right]$, and $\Theta_{q}=\left[\theta_{q, 1} \theta_{q, 2} \cdots \theta_{q, N_{\mathrm{P}}-1}\right]$. For the CRLB computation, we necessarily evaluate the derivative of $\mathbf{S}_{q, k}$ with respect to each parameter, and we then obtain the following results.

$$
\begin{aligned}
& \mathbf{D}_{q, k}^{(x)}=\frac{\partial \mathbf{S}_{q, k}}{\partial x_{0}}=\frac{\partial \mathbf{a}_{q}\left(\theta_{q, 0}\right)}{\partial x_{0}} g_{q, 0} e^{-j \omega_{q, 0} k}, \\
& \mathbf{D}_{q, k}^{(y)}=\frac{\partial \mathbf{S}_{q, k}}{\partial y_{0}}=\frac{\partial \mathbf{a}_{q}\left(\theta_{q, 0}\right)}{\partial y_{0}} g_{q, 0} e^{-j \omega_{q, 0} k} \\
& \mathbf{D}_{q, k}^{(\Omega)}=\frac{\partial \mathbf{S}_{q, k}}{\partial \Omega_{q}}=-j k \mathbf{A}_{q} \mathbf{G}_{q, 0} \operatorname{diag}\left\{\mathbf{R}_{q, 0}^{k}\right\}, \\
& \mathbf{D}_{q, k}^{(\mathbf{g})}=\left[\frac{\partial \mathbf{S}_{q, k}}{\partial \Re\left(\mathbf{g}_{q}\right)} \frac{\partial \mathbf{S}_{q, k}}{\partial \Im\left(\mathbf{g}_{q}\right)}\right]=\left[\mathbf{A}_{q} \operatorname{diag}\left\{\mathbf{R}_{q, 0}^{k}\right\} j \mathbf{A}_{q} \operatorname{diag}\left\{\mathbf{R}_{q, 0}^{k}\right\}\right],
\end{aligned}
$$

and

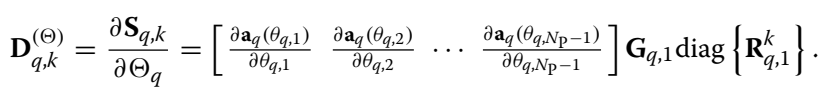

Let $\mathbf{S}_{k}$ denote the column vector of complete observation data for the $k$-th subcarrier:

$$
\mathbf{S}_{k}=\left[\begin{array}{llll}
\mathbf{S}_{1, k}^{\mathrm{T}} & \mathbf{S}_{2, k}^{\mathrm{T}} & \cdots & \mathbf{S}_{Q, k}^{\mathrm{T}}
\end{array}\right]^{\mathrm{T}}
$$

Also, let $\mathbf{D}_{q, k}^{(*)}=\left[\mathbf{D}_{q, k}^{(\Omega)} \mathbf{D}_{q, k}^{(\mathbf{g})} \mathbf{D}_{q, k}^{(\Theta)}\right]$, and $\mathbf{E}_{k}=\partial \mathbf{S}_{k} / \partial \mathbf{u}$. Thus,

$$
\mathbf{E}_{k}=\left[\begin{array}{llllll}
\mathbf{D}_{1, k}^{(x)} & \mathbf{D}_{1, k}^{(y)} & \mathbf{D}_{1, k}^{(*)} & 0 & \cdots & 0 \\
\mathbf{D}_{2, k}^{(x)} & \mathbf{D}_{2, k}^{(y)} & 0 & \mathbf{D}_{2, k}^{(*)} & 0 & \vdots \\
\vdots & \vdots & \vdots & 0 & \ddots & 0 \\
\mathbf{D}_{Q, k}^{(x)} & \mathbf{D}_{Q, k}^{(y)} & 0 & \cdots & 0 & \mathbf{D}_{Q, k}^{(*)}
\end{array}\right] .
$$

Then, based on [19], the Fisher information matrix for the parameter vector $\mathbf{u}$ can be computed by

$$
\mathbf{I}=\sum_{\forall k} \frac{2}{\sigma^{2}} \Re\left(\mathbf{E}_{k}^{\mathrm{H}} \mathbf{E}_{k}\right),
$$

where $(\cdot)^{\mathrm{H}}$ denotes the matrix conjugate and transpose, and $\sigma^{2}$ is the variance of $w_{q, k, m}$. Then, the CRLB for the location error can be computed by

$$
\mathrm{CRLB}=\left[\mathbf{I}^{-1}\right]_{1,1}+\left[\mathbf{I}^{-1}\right]_{2,2},
$$

where $[\mathbf{K}]_{i, j}$ denotes the $(i, j)$ element of matrix $\mathbf{K}$.

\section{Numerical results}

\subsection{Assumptions}

To gain fundamental insights on the system requirements and design principles, we obtain numerical results specific to the following assumptions. 


\subsubsection{Signal model}

The following are assumed for the signal model. An antenna gain of $0 \mathrm{~dB}$ is assumed for the $\mathrm{MD}$ antenna as well as for each array element of a BS. The variance of $w_{q, k, m}$ depends only on the system noise, i.e., $E\left[\left|H_{q, k, m}-w_{q, k, m}\right|^{2}\right] / E\left[\left|w_{q, k, m}\right|^{2}\right]$ equals the average signal-to-noise ratio per OFDM subcarrier. The noise figure of the OFDM receiver is $4 \mathrm{~dB}$. The number of antennas in an antenna array is a constant, i.e., $M_{q}=M, \forall q$. For the OFDM signal, the center frequency is $5.25 \mathrm{GHz}$, the subcarrier spacing $\Delta$ follows that employed in [20], i.e., $\Delta=312.5 \mathrm{kHz}$, and the set of used subcarrier indexes is $\{k \mid-K \leq k \leq K\}$, where $K$ is an integer controlling the bandwidth. We consider the bandwidth without accounting a guard band, i.e., $B=(2 K+1) \Delta=(0.625 K+0.3125) \mathrm{MHz}$.

It should be noted that the total bandwidth $B_{\mathrm{T}}$ practically allocated for the OFDM system is bigger than $B$. This is because guard bands are necessarily allocated to avoid interference from system of nearby spectrum. The guard bands may be implemented by having blocks of subcarriers with zero amplitudes at both ends of the active spectrum. Hence, $B_{\mathrm{T}}=\left(N_{\mathrm{u}}+N_{\mathrm{z}}\right) \Delta$, where $N_{\mathrm{u}}$ is the number of used subcarriers $2 K+1$, and $N_{\mathrm{z}}$ is the number of zero amplitude subcarriers. Then, we may note that while both the guard band and guard interval are necessarily allocated to deal with interference, they necessarily reduce the spectrum efficiency. Assume that the guard interval corresponds to cyclic prefix of $N_{c}$ sample, where the sampling rate is the nominal one for OFDM, i.e., $1 / B_{\mathrm{T}}$. Then, by the proper anticipation of guard band and interval, the average rate of effectively carried information symbols is down-scaled by $N_{\mathrm{u}} /\left(N_{\mathrm{u}}+N_{\mathrm{z}}+N_{\mathrm{c}}\right)$ as noted in [21].

\subsubsection{Geometrical models}

A linear array with half-wavelength spacing between elements is assumed for each BS antenna array. Accordingly, the following geometrical reference model is applied. Cartesian coordinates $\left(x_{0}, y_{0}\right)$ and $\left(x_{q}, y_{q}\right)$ are used for referring to the location of respectively the MD and the array center of the $q$-th BS. The relation between the two locations is represented as shown in Fig. 1 by a polar coordinate $\left(r_{q}, \theta_{q, 0}\right)$. The orientation of an antenna array is represented by the normal looking direction of the array defined as follows. The normal looking direction is determined by first setting the phase shifts of all the antenna elements to be zero and then determining the main radiation direction that is closest to the direction of arrival of the MD. We use $\gamma_{q}$ to represent the normal looking direction for the $q$-th BS, as a counterclockwise angle relative to the $x$-axis. For example, if the placement line of the $q$-th BS antenna array is on the $x$-axis in Fig. $1, \gamma_{q}$ will be $\pi / 2$. Assuming $r_{q}$ is much greater than the spacing between

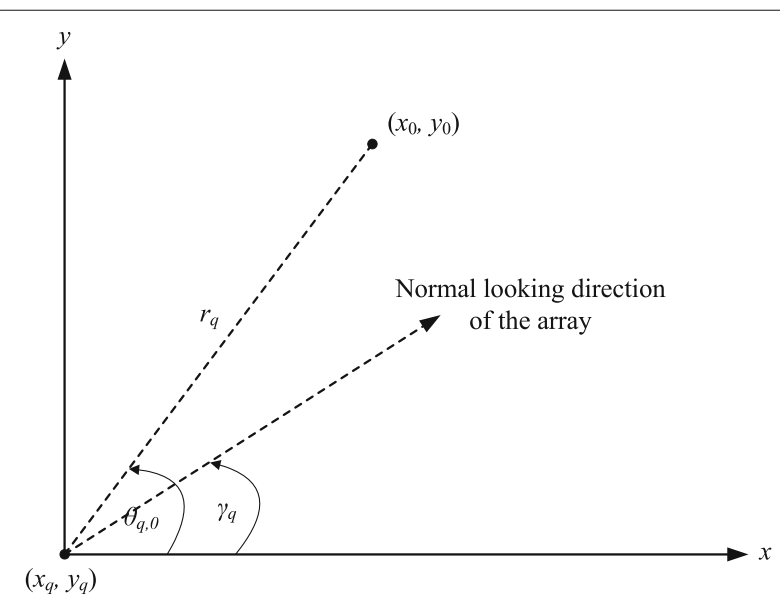

Fig. 1 Illustration of geometrical reference model. Illustration of geometrical reference model of $\left(x_{0}, y_{0}\right),\left(x_{q}, y_{q}\right),\left(r_{q}, \theta_{q, 0}\right)$, and $\gamma_{q}$

the antenna array elements, then $\phi_{q, n, m}, \forall(q, n, m)$ can be approximated by

$$
\phi_{q, n, m}=\pi(m-(M+1) / 2) \sin \left(\theta_{q, n}-\gamma_{q}\right) .
$$

Thus,

$$
\frac{\partial \mathbf{a}_{q}\left(\theta_{q, n}\right)}{\partial \theta_{q, n}}=-j \pi \cos \left(\theta_{q, n}-\gamma_{q}\right) \operatorname{diag}\{m-(M+1) / 2\}_{m=1}^{M} \mathbf{a}_{q}\left(\theta_{q, n}\right),
$$

$$
\begin{aligned}
\frac{\partial \mathbf{a}_{q}\left(\theta_{q, 0}\right)}{\partial x_{0}}= & j \pi r_{q}^{-1} \sin \left(\theta_{q, 0}\right) \cos \left(\theta_{q, 0}-\gamma_{q}\right) \\
& \operatorname{diag}\{m-(M+1) / 2\}_{m=1}^{M} \mathbf{a}_{q}\left(\theta_{q, 0}\right),
\end{aligned}
$$

and

$$
\begin{aligned}
\frac{\partial \mathbf{a}_{q}\left(\theta_{q, 0}\right)}{\partial y_{0}}= & -j \pi r_{q}^{-1} \cos \left(\theta_{q, 0}\right) \cos \left(\theta_{q, 0}-\gamma_{q}\right) \\
& \operatorname{diag}\{m-(M+1) / 2\}_{m=1}^{M} \mathbf{a}_{q}\left(\theta_{q, 0}\right) .
\end{aligned}
$$

The performance results will be obtained by averaging the computed CRLBs over various values of the parameter vector $\mathbf{u}$. Figure 2 shows the geometrical assumptions, on which the variation of $\mathbf{u}$ is based. It shows that the region of interest for localization is a square with area of $400 \mathrm{~m}^{2}$, there is a BS located at each corner, and the normal looking directions of the four arrays are pointed towards the region center.

\subsubsection{Multipath propagation model}

Radio wave propagation is subjected to multipath characteristics, which depend on the environment. In this paper, the multipath characteristics are varied, respecting density of the multipaths and relationship between the powers of direct and reflection paths, for investigation of their impacts on the CRLB performance. In this regard, the following statistical model is employed. 


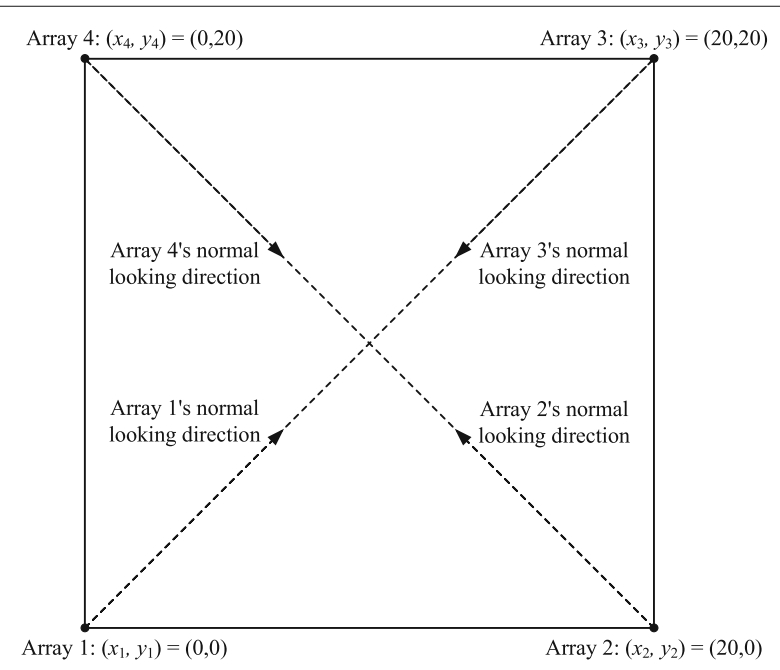

Fig. 2 Illustration of geometrical simulation assumptions. The $400-\mathrm{m}^{2}$ region of interest for localization and the normal looking directions of the antenna arrays of four BSs located at the corners

The direct path The direct path parameters for the $q$-th BS consist of only $g_{q, 0}$ and $\omega_{q, 0}$, because $\theta_{q, 0}$ can be determined form $x_{0}$ and $y_{0}$. In this paper, $\angle g_{q, 0}$ is drawn from a uniform distribution over $[0,2 \pi)$, and $\left|g_{q, 0}\right|$ is obtained, based on the free-space path loss model. Assume the variance of $w_{q, k, m}$ is $\sigma^{2}=1$. Also, recall that the gain of each antenna is $0 \mathrm{~dB}$. Thus,

$$
\left|g_{q, 0}\right|^{2}=P_{\mathrm{t}}\left(\frac{c}{4 \pi f_{\mathrm{c}} r_{q}}\right)^{2} /\left(\kappa T_{\mathrm{e}} B\right),
$$

where $P_{\mathrm{t}}$ is the MD transmitted power, $c$ is the light speed, $f_{\mathrm{c}}=5.25 \mathrm{E}+9 \mathrm{~Hz}$ as assumed, $\kappa$ is the Boltzmann constant, and $T_{\mathrm{e}}$ is the effective noise temperature of the BS receiver. For the value of $\omega_{q, 0}$, we set $t_{q, 0}=0$, based on the discussion in Section 2.

The reflection paths In this paper, $\angle g_{q, n}$ and $\theta_{q, n}, \forall n>0$ are independently drawn from a uniform distribution over $[0,2 \pi)$. In addition, $\left|g_{q, n}\right|$ and $\omega_{q, n}, n>0$ are set to obey

$$
\frac{\left|g_{q, n-1}\right|^{2}}{\left|g_{q, n}\right|^{2}}=\rho
$$

and

$$
t_{q, n}-t_{q, n-1}=\delta
$$

where $\rho$ reflects a constant power-decay condition, and $\delta^{-1}$ reflects the multipath density. The magnitude of a reflection path is not modeled to have a random characteristic, as it is treated as a controlled variable of the study. However, as the reflection paths are delayed relative to the direct path and their complex amplitudes have identical and independent random phases, the frequency response over the frequency band of the signal has a random frequency-selective fading characteristic. In this regard, we may note from (1) that the CFR for a specific subcarrier is a summation of independent complex zero mean circularly symmetric random variables. Hence, for large $N_{\mathrm{P}}$, the frequency response over a subcarrier could exhibit frequency flat-fading characteristics with Rayleigh or Rician distribution, the common characteristics in practical radio propagation environments [22]. The Rayleigh distribution could occur when $\left|g_{q, 0}\right|^{2} \ll \sum_{n=1}^{N_{\mathrm{P}}-1}\left|g_{q, n}\right|^{2}$, which is possible with $\rho \approx 1$. This is because when $\rho=1$, we have $\left|g_{q, n}\right|=\left|g_{q, 0}\right|$ for all $n>0$ and accordingly the Rician $K$ factor [22] is $1 /\left(N_{\mathrm{P}}-1\right) \approx 0$. On the other hand, the Rician distribution could occur when $\left|g_{q, 0}\right|^{2} \gg \sum_{n=1}^{N_{\mathrm{P}}-1}\left|g_{q, n}\right|^{2}$, which is possible with $\rho \gg 1$.

\subsection{Discussion}

The mean square error (MSE) performance results presented here are obtained by averaging the computed CRLBs over various values of the parameter vector $\mathbf{u}$. In this regard, the MD is uniformly and randomly located in the $400-\mathrm{m}^{2}$ region, except the locations that are close to the center of an antenna array by less than $1 \mathrm{~m}$. The performance will be presented for $P_{\mathrm{t}}=-20 \mathrm{dBm}$, respecting the infrastructure conditions $(B$ and $M)$ and the radio propagation characteristics $\left(\rho, \delta\right.$, and $\left.N_{\mathrm{P}}\right)$. The performance respecting $P_{\mathrm{t}}$ is not presented, because it is obvious that the MSE-versus- $P_{\mathrm{t}}$ curve in this case is a line with a slope of $-10 \mathrm{~dB} /$ decade. Note also that a study of the performance respecting geometry may be useful in determining the positions of BS antenna arrays [23]. Such study is beyond the scope of our work, and we believe it merits further investigation.

Figures 3 and 4 show the MSE performance in relation to $B$ and $M$, for $\delta=10 \mathrm{~ns}, \rho=0 \mathrm{~dB}$, and $N_{\mathrm{P}}=5$. Note that changing $M$ affects the quantity of observation

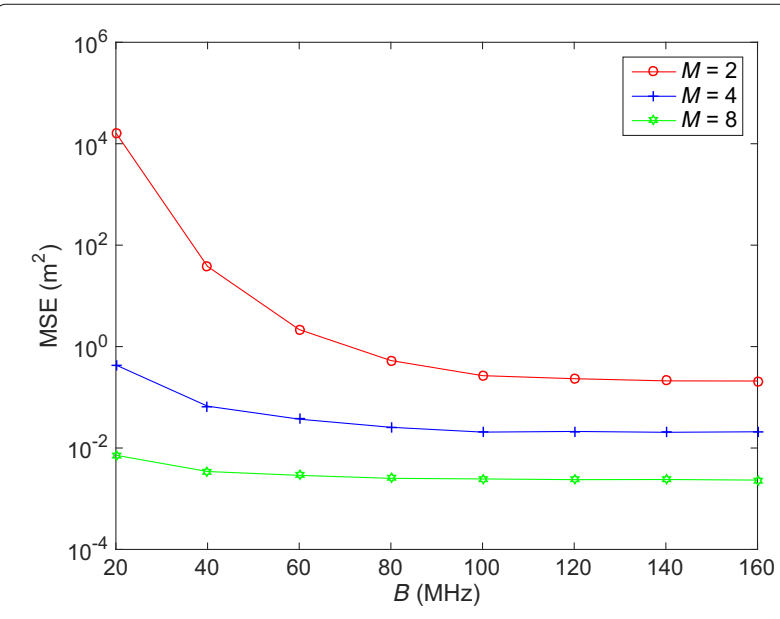

Fig. 3 Numerical result 1. MSE versus $B$ with $M$ as a parameter, for $\delta=10 \mathrm{~ns}, \rho=0 \mathrm{~dB}$, and $N_{p}=5$ 


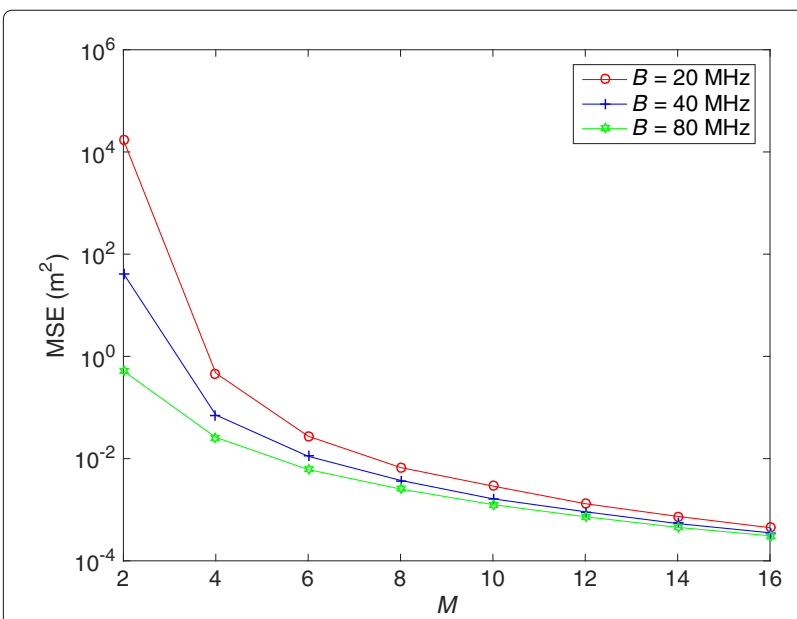

Fig. 4 Numerical result 2. MSE versus $M$ with $B$ as a parameter, for $\delta=10 \mathrm{~ns}, \rho=0 \mathrm{~dB}$, and $N_{\mathrm{p}}=5$

data and accordingly the number of rows of $E_{k}$ that equals $Q M$. Also, changing $B$ affects the quantity of observation data and accordingly the number of summands in (16) that equals $2 K+1$. In addition, by changing $B$, we must recompute $g_{q, n}, \forall(q, n)$, according to (22) and (23), for use in the construction of new $E_{k}$. Figure 3 shows that the MSE may be reduced by increasing $B$, but there is a limit where the MSE cannot be reduced further. According to the figure, that limit is found to be reachable at smaller $B$ for larger $M$. That limit is also found to depend on $M$, as the limits for $M=2,4$, and 8 are respectively about $0.2094,0.0201$, and $0.0023 \mathrm{~m}^{2}$. Figure 4 shows that the MSE may be reduced by increasing $M$. However, as $M$ becomes larger, the MSE always decreases while the importance of $B$ becomes lesser, as the MSE limit discussed above is being reached. Hence, the above observations may be summarized as follows. The MSE performance can be generally improved by increasing either $B$ or $M$, but increasing $B$ can only reduce the MSE to a limit depending on $M$. Increasing $M$ alone can push the MSE to the limit, which is also smaller as $M$ increases. In addition, with a larger $M$, a smaller $B$ would be required for the MSE to approach the limit.

Figure 5 shows the MSE performance in relation to $N_{\mathrm{P}}$ and $M$, for $B=40 \mathrm{MHz}, \delta=10 \mathrm{~ns}$, and $\rho=0 \mathrm{~dB}$, which includes the free-space results $\left(N_{\mathrm{P}}=1\right)$. Note that changing $N_{P}$ affects the number of parameters for estimation and accordingly the number of columns of $E_{k}$ that equals $\left(4 N_{\mathrm{P}}-1\right) Q+2$. Interestingly, it should be first noted that the free-space results in Fig. 5 and the MSE limits in Fig. 3 are same. This suggests that the MSE limit previously discussed corresponds to the performance virtually obtained from free-space channel. Hence, in this paper, we will refer to the free-space channel result as the free-space limit. This limiting characteristic of the MSE is consistent with the following principles. First, note from

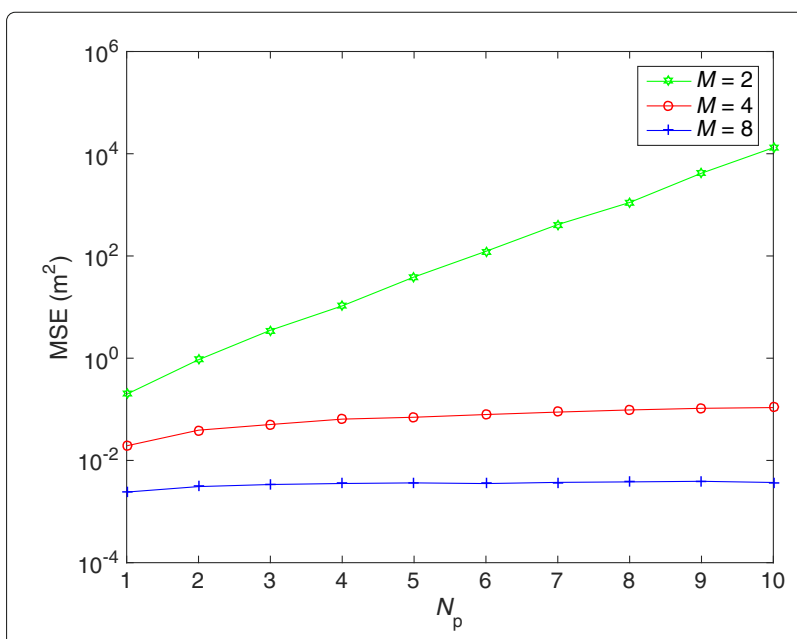

Fig. 5 Numerical result 3. MSE versus $N_{p}$ with $M$ as a parameter, for $B=40 \mathrm{MHz}, \delta=10 \mathrm{~ns}$, and $\rho=0 \mathrm{~dB}$

(1) that the contribution of a particular path is a complex sinusoidal function of $k$ with a particular frequency depending on the path delay. In this regard, we should note that direct-path AOA estimation basically requires separating the direct-path contribution from reflectionpath contributions, where large $B$ is of great help. This is because of that the key difference between the paths is the sinusoidal frequency and that a less ambiguous result on such frequency determination basically requires observing the CFR over a larger range of $k$. Then, once $B$ is large enough for perfect path separation, further increasing $B$ while retaining the total transmitted power as assumed here is not seen to further improve the localization performance. This indicates that AOA estimation performance for a perfectly separated path depends on the total transmitted signal power, regardless of the signal bandwidth. Hence, increasing $B$ by more spreading of the fixed transmitted power over the spectrum can only help the MVUE separate the multipaths, which eventually pushes the performance to approach the free-space limit. Figure 5 also shows that the logarithm of the MSE rises almost linearly with $N_{\mathrm{P}}$ for $M=2$, while for other $M$, the MSE remains almost constant even if $N_{\mathrm{P}}$ is close to 10 . The results for $N_{\mathrm{P}}=5$ in Fig. 5 are consistent with the results of the same case, i.e., $B=40 \mathrm{MHz}$, in Fig. 3. The results of the mentioned case from both figures consistently indicate that the MSE is far from the free-space limit for $M=2$, while for other $M$, the MSE is already close to the free-space limit. Hence, if the MSE is already close to the free-space limit due to an appropriate provision of $B$ and $M$ to deal with the multipath environment, the MVUE will be robust against variation of $N_{\mathrm{P}}$. Otherwise, the corresponding MSE will rise exponentially with $N_{P}$.

Figure 6 shows the MSE performance in relation to $N_{\mathrm{P}}$ and $B$, for $M=2, \delta=10 \mathrm{~ns}$, and $\rho=0 \mathrm{~dB}$. 


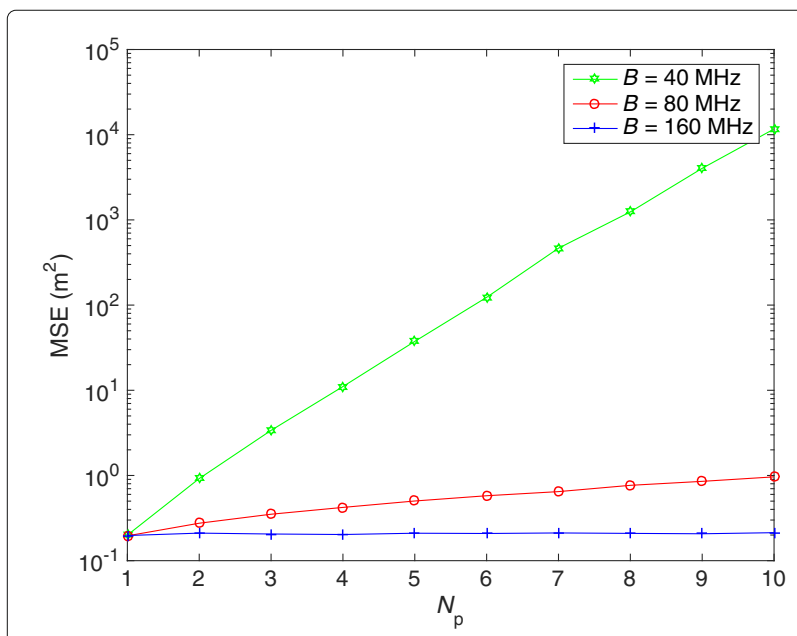

Fig. 6 Numerical result 4. MSE versus $N_{\mathrm{p}}$ with $B$ as a parameter, for $M=2, \delta=10 \mathrm{~ns}$, and $\rho=0 \mathrm{~dB}$

The figure provides same observations as previously discussed, regarding the characteristics of MSE against $N_{\mathrm{P}}$ and impact of increasing $B$ on the MSE performance for multipath environments. Interestingly, it also shows that the free-space results do not depend on $B$. Theoretically, this may be justified by the following considerations. For $N_{\mathrm{P}}=1$, (1) becomes $H_{q, k, m}=g_{q, 0} e^{-j\left(\omega_{q, 0} k+\phi_{q, 0, m}\right)}+$ $w_{q, k, m}$. The AOA-based localization basically relies on the estimation of $\phi_{q, 0, m}, \forall(q, m)$, in which the AOA information is contained. As a result of [24], we note that the CRLB of $\phi_{q, 0, m}$ based on the $(2 K+1)$ sample observation of $H_{q, k, m}$ is inversely proportional to $\left|g_{q, 0}\right|^{2}(2 K+1)$ for large $K$. Recall that $B=(2 K+1) \Delta$, and $\left|g_{q, 0}\right|^{2} B$ is proportional to $P_{\mathrm{t}}$ according to (22). Therefore, $\left|g_{q, 0}\right|^{2}(2 K+1)$ is also proportional to $P_{\mathrm{t}}$. In addition, the CRLB of $\phi_{q, 0, m}$ depends on $P_{\mathrm{t}}$, instead of $B$. Note also that by first-order approximation, the association between a small error of $\phi_{q, 0, m}$ and the resulting localization error is approximately linear. Therefore, the free-space localization CRLB results also depend on $P_{\mathrm{t}}$, instead of $B$.

Finally, regarding comparison between the impacts of $B$ and $M$ on the MSE performance, we note from Figs. 3, 4, 5 and 6 that doubling $M$ gives greater performance benefit than doubling $B$ for all cases observable from the figures. This should come from the following principles of AOAbased localization using (1). Increasing $B$ while maintaining $P_{\mathrm{t}}$ only helps the MVUE separate the multipaths. On the other hand, increasing $M$ not only provides more observation data that help multipath separation but also improves AOA sensitivity and therefore the free-space limit.

Figure 7 shows the MSE performance in relation to $\delta$ and $M$, for $B=80 \mathrm{MHz}, \rho=0 \mathrm{~dB}$, and $N_{\mathrm{P}}=10$, while Fig. 8 shows the MSE performance in relation to $\delta$

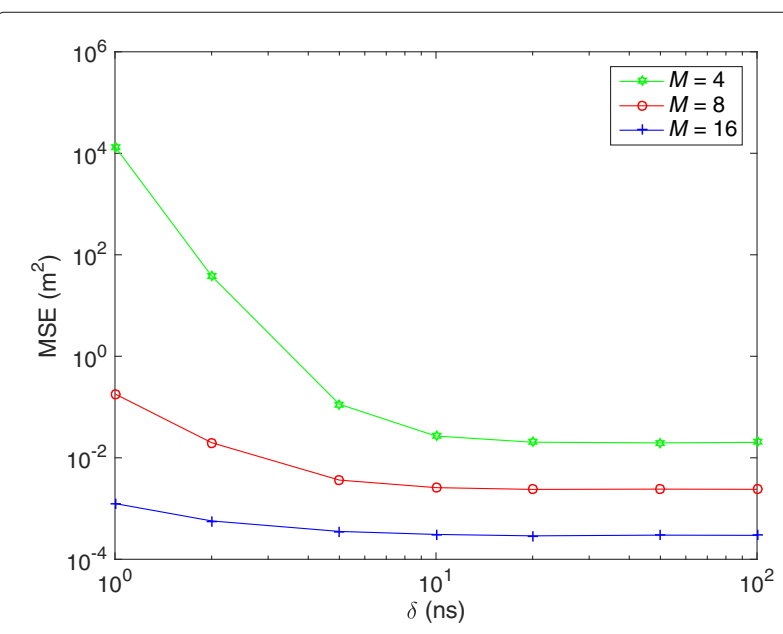

Fig. 7 Numerical result 5. MSE versus $\delta$ with $M$ as a parameter, for $B=80 \mathrm{MHz}, \rho=0 \mathrm{~dB}$, and $N_{\mathrm{p}}=10$

and $B$, for $M=4, \rho=0 \mathrm{~dB}$, and $N_{\mathrm{P}}=10$. Note that changing $\delta$ does not affect either the size of $E_{k}$ or the number of summands in (16), because it does not affect either quantity of observation data or the number of parameters for estimation. By changing $\delta$, we only recompute $\omega_{q, n}$, $n>0, \forall q$ to obey (24) for use in the construction of new $E_{k}$. Below are the reported various observations from the figures. The multipath density has a profound impact on the MSE performance, as the performance tends to get severely worse than the free-space limit when $\delta$ is lower than a certain boundary value that can be reduced by increasing $B$ or $M$. For example, it can be seen from Fig. 7 that for $M=4$, the MSE starts to get worse than the free-space limit when the $\delta$ is decreasing below a value between 5 and $10 \mathrm{~ns}$, while for a larger $M$, such characteristic occurs at a smaller $\delta$. It can be also seen from Fig. 8

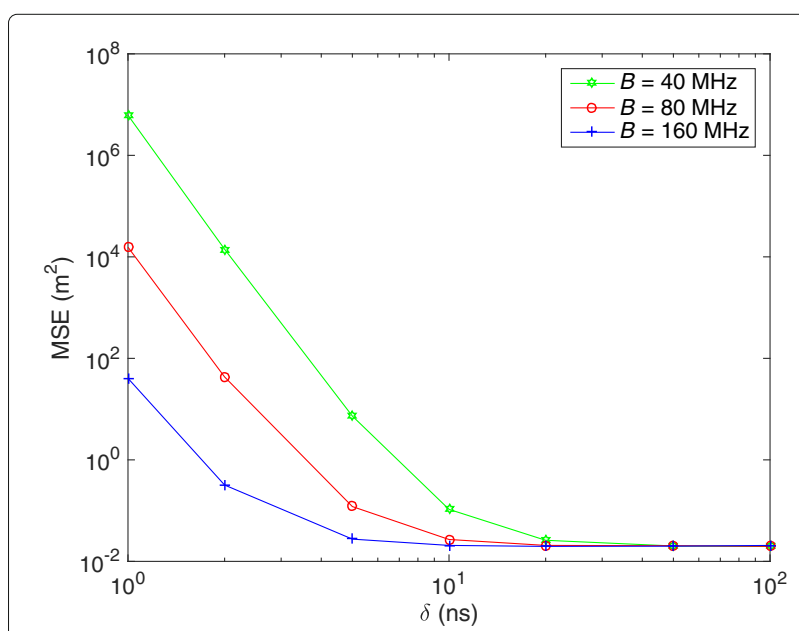

Fig. 8 Numerical result 6. MSE versus $\delta$ with $B$ as a parameter, for $M=4, \rho=0 \mathrm{~dB}$, and $N_{p}=10$ 
that for $B=40 \mathrm{MHz}$, the MSE starts to get worse than the free-space limit when the $\delta$ is decreasing below a value between 10 and $20 \mathrm{~ns}$, while for a larger $B$, such characteristic occurs at a smaller $\delta$. It may be also noted from Fig. 7 that in addition to reducing the boundary value, provision for a larger $M$ also reduces the rate of deterioration when the $\delta$ decreases below beyond the boundary value. Again, regarding comparison between the impacts of $B$ and $M$ on the MSE performance, we see that increasing $M$ provides greater performance benefit.

Figure 9 shows the MSE performance in relation to $\rho$ and $M$, for $B=80 \mathrm{MHz}, \delta=2 \mathrm{~ns}$, and $N_{\mathrm{P}}=10$. Note that changing $\rho$ does not affect either the size of $E_{k}$ or the number of summands in (16). By changing $\rho$, we only recompute $g_{q, n}, n>0, \forall q$ to obey (23) for use in the construction of new $E_{k}$. The figure shows that all curves are essentially flat, and therefore, the MSE seems to be independent of $\rho$. Comparing the MSE results in Fig. 9 and the free-space limits observable from Fig. 7, we see that the MSE at $\delta=2 \mathrm{~ns}$ is farthest to the free-space limit when $M=4$, while it is nearest when $M=16$. The flat characteristic of the curves in Fig. 9 exists, no matter how far from the free-space limit the MSE is. This may sound surprising, because the channel at $\rho=20 \mathrm{~dB}$ is very much alike a free-space channel, and for this condition, one can easily design an algorithm that performs very close to the free-space limit. An algorithm that simply treats the channel as a free-space channel should be able to achieve such performance, because that insignificant model mismatch should only cause insignificant bias to the estimation result. This also means that when the modeled reflection paths are weak, the MVUE could perform surprisingly as poor as the case where the paths are strong. Such behavior of the MVUE, which embraces all modeled paths into its idealized unbiased estimation task, will be

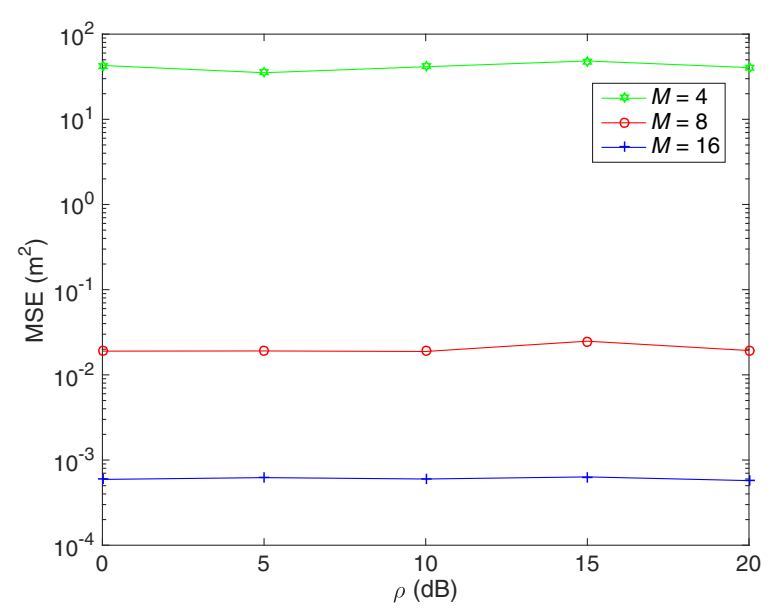

Fig. 9 Numerical result 7. MSE versus $\rho$ with $M$ as a parameter, for $B=80 \mathrm{MHz}, \delta=2 \mathrm{~ns}$, and $N_{\mathrm{p}}=10$ mathematically justified soon in this section. Hence, we suggest that reflection paths with insignificant amplitude should not be included explicitly in the channel model. This is to maintain that the CRLB may be also meaningful in a study of biased estimation algorithms.

We may claim more strictly from the results in Fig. 9 that the localization performance of the MVUE is independent of the strength of a reflection path. This can be mathematically justified by the following considerations. Note that the gain of a particular reflection path appears as a constant factor consistently for all non-zero elements along only two columns of $E_{k}$. For a specific $g_{q, n}, n>0$, such two columns correspond to respectively the $(n+1)$-th column of $\mathbf{D}_{q, k}^{(\Omega)}$ and the $n$-th column of $\mathbf{D}_{q, k}^{(\Theta)}$. The indexes of them within $E_{k}$ are always greater than two, because they all belong to $\mathbf{D}_{q, k}^{(*)}$ in (15). Therefore, by scaling the value of the $g_{q, n}$ with a real factor, $E_{k}$ can be recomputed accordingly by post-multiplying itself with a real constant diagonal matrix, where all diagonal elements are equal to one except the elements associated with the two indexes. The inverse of the Fisher information matrix can then be recomputed by pre- and post-multiplying itself with the inverse of that diagonal matrix. Such operation will not affect the localization performance of the MVUE computed by (17), because the two indexes are always greater than two.

We have been realizing from the results of Figs. 7 and 8 that the multipath density reflected by $\delta$ is crucial to the effectiveness of the infrastructure reflected by $B$ and $M$. However, we consider that care may be only required for the second path that has delay closest to the direct path, because it contributes most to the difficulty of separating the direct path from other paths. Thus, it may be more interesting to investigate the case of channels where the path delay rule in (24) is replaced by

$$
t_{q, n}-t_{q, n-1}=\left\{\begin{array}{l}
\delta_{0}, \text { if } n=1 \\
\delta_{1}, \text { otherwise. }
\end{array}\right.
$$

Figure 10 shows the MSE performance in relation to $\delta_{1}$ and $B$, for $M=4, \rho=0 \mathrm{~dB}, N_{\mathrm{P}}=10, \delta_{0}=10$ ns, and $\delta_{1} \leq 10 \mathrm{~ns}$, which can then be compared with the cases $\delta_{0}=\delta_{1} \leq 10$ ns presented in Fig. 8. We can then see that the performance degradation due to moving from $\delta_{0}=\delta_{1}=10$ ns to $\delta_{0}=10$ ns with $\delta_{1}=1$ ns is much less than the degradation due to moving from the same original condition to $\delta_{0}=\delta_{1}=1 \mathrm{~ns}$. Hence, allowing the reflection paths to be close in delay between themselves could not degrade the performance so much, compared to allowing significant reflection paths to be close in delay to the direct path. It is then very important to give much care for each significant reflection path with delay close to that of the direct path. Note that a position of scatterer that could produce such a reflection path can only be around the straight line connecting the 


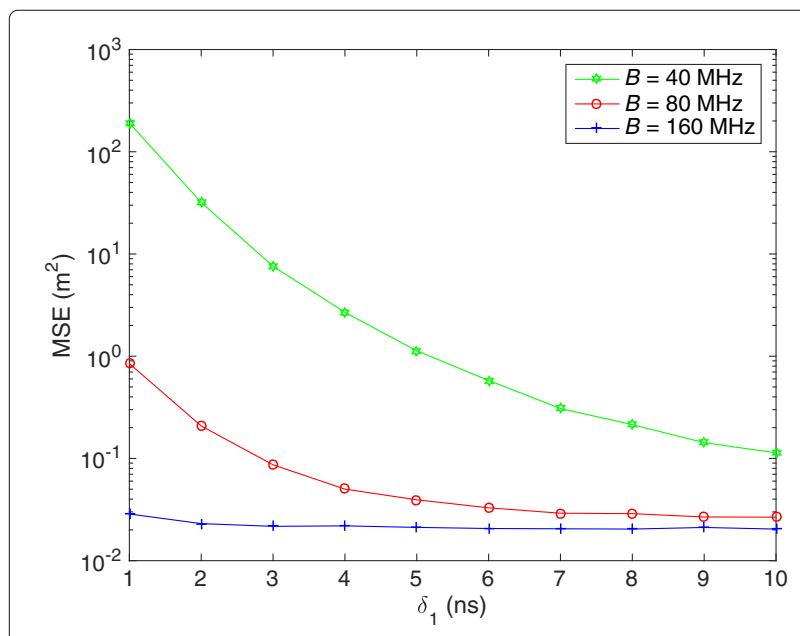

Fig. 10 Numerical result 8. MSE versus $\delta_{1}$ with $B$ as a parameter, for $M=4, \rho=0 \mathrm{~dB}, N_{\mathrm{p}}=10$, and $\delta_{0}=10 \mathrm{~ns}$

MD and the BS. For such a position, we may further note that everyone around the BS is most cumbersome. This is because the corresponding reflection path AOA can be considerably different from the direct-path AOA, and then, the reflection path needs to be modeled explicitly as having such different AOA but similar delay to that of the direct path. On the other hand, for such a scatterer position rather than those around the BS, the corresponding reflection path AOA cannot be considerably different from the direct-path AOA. Accordingly, by neglecting the seen insignificant difference on both the delay and AOA, the reflection path can be approximately modeled by merging itself with the direct path based on only complex path-amplitude addition. Therefore, it is important to avoid the most cumbersome scatterers by ensuring that the BS antenna arrays are carefully placed not close to any significant reflective materials, and in some cases, using radio-absorptive materials may be helpful.

\section{Conclusions}

We studied the problem of AOA-based localization using MIMO-OFDM channel state information as observation data. In particular, we derived a method to compute the CRLB which is a fundamental limit on the localization MSE and applied it to obtain fundamental insights into the problem. We found that the CRLB is independent of the strength of a reflection path. Therefore, we suggested that reflection paths with insignificant amplitude should not be included explicitly in the channel model. Provided that the mobile device-transmitted signal power is fixed, the following may be concluded from our investigation. The MSE localization performance can be improved by increasing the transmitted signal bandwidth $B$ alone. However, there is a limit where further increasing $B$ cannot further improve the performance. The limit is found to equal the performance virtually obtained from the free-space radio propagation assumption. The performance can be considerably improved also by increasing the base station antenna array size $M$ alone, as, when $M$ increases, not only does the free-space limit decrease but also the MSE gets closer to the decreasing limit. In particular, scaling $M$ always has greater impact on the MSE than scaling $B$. Provided that the MSE is close to the free-space limit, the optimum unbiased estimator will be robust to variation on the number of multipaths $N_{\mathrm{P}}$. Otherwise, the MSE will rise exponentially with $N_{\mathrm{P}}$. The multipath density that is inversely proportional to $\delta$ has a profound impact on the MSE performance, as the performance tends to get severely worse than the free-space limit when the density is higher than a value proportional to $B$ and $M$. Finally, when significant reflection paths with AOAs very different from that of the direct path have similar delays to that of the direct path, the localization performance could be severely degraded.

\section{Acknowledgements}

The authors would like to thank the anonymous reviewers for their constructive comments, which helped a lot to improve the presentation of this paper.

\section{Funding}

Not applicable.

\section{Authors' contributions}

Both authors contributed to the searching of the literature. TD contributed to theoretical analysis. Both authors contributed to the design and implementation of computer simulation experiments and interpretation of the results. Both authors have read and approved the final manuscript.

\section{Competing interests}

The authors declare that they have no competing interests.

\section{Publisher's Note}

Springer Nature remains neutral with regard to jurisdictional claims in published maps and institutional affiliations.

Received: 9 January 2017 Accepted: 4 August 2017

Published online: 03 October 2017

\section{References}

1. H Liu, H Darabi, P Banerjee, J Liu, Survey of wireless indoor positioning techniques and systems. IEEE Trans. Syst. Man Cybern. Part C Appl. Rev. 37(6), 1067-1080 (2007)

2. Z Farid, R Nordin, M Ismail, Recent advances in wireless indoor localization techniques and system. J. Comput. Netw. Commun. 2013(185138), 1-12 (2013)

3. KYu, I Sharp, YJ Guo, Ground-based wireless positioning, 1st edn. (Wiley, West Sussex, 2009)

4. Y Zhao, K Liu, Y Ma, Z Li, An improved k-NN algorithm for localization in multipath environments. EURASIP J. Wirel. Commun. Netw. 2014(208), $1-10$ (2014)

5. MB Zeytinci, V Sari, FK Harmanci, E Anarim, M Akar, Location estimation using RSS measurements with unknown path loss exponents. EURASIP J. Wirel. Commun. Netw. 2013(178), 1-14 (2013)

6. P Bahl, VN Padmanabhan, in IEEE INFOCOM. RADAR: an in-building RF-based user location and tracking system (The Institute of Electrical and Electronics Engineers (IEEE), Piscataway, 2000), pp. 775-784 
7. M Youssef, A Agrawala, in Proceedings of the 3rd International Conference on Mobile Systems, Applications, and Services. The Horus WLAN Location Determination System (Association for Computing Machinery (ACM), New York, 2005), pp. 205-218

8. KWu, J Xiao, Y Yi, D Chen, X Luo, LM Ni, CSI-based indoor localization. IEEE Trans. Parallel Distrib. Syst. 24(7), 1300-1309 (2013)

9. T Demeechai, P Kukieattikool, T Ngo, T-G Chang, Localization based on standard wireless lan infrastructure using mimo-ofdm channel state information. EURASIP J. Wirel. Commun. Netw. 2016(146), 1-16 (2016)

10. NB Rejeb, I Bousnina, MBB Salah, A Samet, Joint mean angle of arrival, angular and Doppler spreads estimation in macrocell environments. EURASIP J. Adv. Signal Process. 2014(133), 1-10 (2014)

11. SO Al-Jazzar, A Muchkaev, A Al-Nimrat, M Smadi, Low complexity and high accuracy angle of arrival estimation using eigenvalue decomposition with extension to 2D AOA and power estimation. EURASIP J. Wirel. Commun. Netw. 2011(123), 1-13 (2011)

12. Y Shen, MZ Win, Fundamental limits of wideband localization: Part I: a general framework. IEEE Trans. Inf. Theory. 56(10), 4956-4980 (2010)

13. H Godrich, AM Haimovich, RS Blum, Target localization accuracy gain in mimo radar-based systems. IEEE Trans. Inf. Theory. 56(6), 2783-2803 (2010)

14. K Witrisal, E Leitinger, S Hinteregger, P Meissner, Bandwidth scaling and diversity gain for ranging and positioning in dense multipath channels. IEEE Wirel. Commun. Lett. 5(4), 396-399 (2016)

15. H Minn, N Al-Dhahir, Optimal training signals for MIMO OFDM channel estimation. IEEE Trans. Wirel. Commun. 5(5), 1158-1168 (2006)

16. HZhu, J Wang, Chunk-based resource allocation in OFDMA systems-part I: chunk allocation. IEEE Trans. Commun. 57(9), 2734-2744 (2009)

17. H Zhu, J Wang, Chunk-based resource allocation in OFDMA systems - part II: Joint chunk, power and bit allocation. IEEE Trans. Commun. 60(2), 499-509 (2012)

18. H Zhu, Radio resource allocation for OFDMA systems in high speed environments. IEEE J. Sel. Areas Commun. 30(4), 748-759 (2012)

19. SM Kay, Fundamentals of statistical signal processing: estimation theory. (Prentice Hall, New Jersey, 1993), p. 47

20. IEEE Std 802.11ac-2013, IEEE Standard for Information Technology-Telecommunications and information exchange between systemsÜlocal and metropolitan area networks-specific requirements, Part 11: wireless LAN medium access control (MAC) and physical layer (PHY) specifications, Amendment 4: enhancements for very high throughput for operation in bands below $6 \mathrm{GHz}$. (The Institute of Electrical and Electronics Engineers, Inc., New York, NY, USA, 2013)

21. SK Chronopoulos, C Votis, V Raptis, G Tatsis, P Kostarakis, in AIP Conference Proceedings 1203. in depth analysis of noise effects in orthogonal frequency division multiplexing systems, utilising a large number of subcarriers (American Institute of Physics (AIP), New York, 2010), pp. 967-972

22. SK Chronopoulos, V Christofilakis, G Tatsis, P Kostarakis, Performance of turbo coded OFDM under the presence of various noise types. Wirel. Pers. Commun. 87(4), 1319-1336 (2016)

23. W Shi, X Qi, J Li, S Yan, L Chen, Y Yu, X Feng, Simple solution to the optimal deployment of cooperative nodes in two-dimensional TOA-based and AOA-based localization system. EURASIP J. Wirel. Commun. Netw. 2017(79), 1-16 (2017)

24. DC Rife, RR Boorstyn, Single-tone parameter estimation from discrete-time observations. IEEE Trans. Inf. Theory. 20(5), 591-598 (1974)

\section{Submit your manuscript to a SpringerOpen ${ }^{\circ}$ journal and benefit from:}

- Convenient online submission

Rigorous peer review

- Open access: articles freely available online

- High visibility within the field

- Retaining the copyright to your article

Submit your next manuscript at $>$ springeropen.com 